\title{
Richtlinien «Abgrenzung von Standardtherapie und experimenteller Therapie im Einzelfall»: definitive Fassung liegt vor
}

Schweizerische Akademie der Medizinischen

Wissenschaften (SAMW)
Spektakuläre Fälle von «experimentellen Therapien», wie z.B. die Bauchspülung mit Methylenblau oder die onkologische Behandlung einer schwerkranken Patientin mit Lipoteichonsäure (LTA) haben öfters zu Schlagzeilen geführt. Gerichtsfälle zeigten, dass die Informationspflicht über den experimentellen Charakter einer Therapie und die in solchen Situationen bestehende erhöhte Sorgfaltspflicht nicht immer genügend wahrgenommen wurden. Dies veranlasste die Stiftung SPO Patientenschutz, im neuen Bundesgesetz über die Forschung am Menschen (HFG) eine entsprechende Regelung zu fordern. Dieser Vorschlag wurde zwar nicht berücksichtigt, das pien können im Rahmen eines Forschungsprojekts angewendet werden. Forschungsuntersuchungen sind demzufolge nicht von der Standardtherapie abzugrenzen, sondern können eine ergänzende Dimension sowohl der Standardbehandlung als auch der experimentellen Behandlung sein.

Der Senat hat eine erste Fassung der Richtlinien im November 2013 zur Vernehmlassung verabschiedet. In der Vernehmlassung sind insgesamt 47, teilweise sehr ausführliche, Stellungnahmen eingetroffen. Mit Ausnahme von zwei Rückmeldungen wurde die Ausarbeitung von Richtlinien in diesem Bereich ausdrücklich begrüsst.

\section{«Die Durchführung einer experimentellen Therapie ist mit erhöhten Sorgfaltspflichten verbunden.»}

\author{
Korrespondenz: \\ lic. iur. Michelle Salathé \\ Schweizerische Akademie der \\ Medizinischen Wissenschaften \\ (SAMW) \\ Petersplatz 13 \\ CH-4051 Basel \\ Tel. 0612699030 \\ m.salathe[at]samw.ch
}

Parlament hat den Bundesrat aber mit einer Motion beauftragt, den Handlungsbedarf abzuklären und allenfalls einen Regelungsvorschlag auszuarbeiten.

Das Thema ist aus Sicht der SAMW wichtig. Experimentelle Therapien bedürfen einer ausreichenden wissenschaftlichen Grundlage, und die Patienten müssen über deren zum Teil unbekannten Risiken und potentiellen Nutzen genügend aufgeklärt werden. Nur so kann die Verletzung grundlegender Prinzipien des Arzt-Patientenverhältnisses vermieden werden. Zur Sensibilisierung der Ärzteschaft hat die SAMW deshalb medizin-ethische Richtlinien zur «Abgrenzung von Standardtherapie und experimenteller Therapie im Einzelfall» ausgearbeitet. Im Laufe der Arbeiten zeigte sich, dass die Herausforderung nicht - wie ursprünglich angenommen - darin besteht, Forschung von Nicht-Forschung abzugrenzen, sondern Kriterien dafür festzulegen, wann eine Therapie vom Standard abgedeckt ist und wann es sich um eine experimentelle Therapie handelt. Sowohl Standardtherapien als auch experimentelle Thera-
Folgende Botschaften der Richtlinien sind zentral:

- Um eine Standardtherapie handelt es sich dann, wenn sich die Therapie auf aktuell gültige fachliche Empfehlungen stützt oder - beim Fehlen solcher Empfehlungen - der allgemein geübten Praxis anerkannter Experten entspricht.

- Eine experimentelle Therapie liegt vor, wenn eine Therapie von der Standardtherapie abweicht bzw. bei Fehlen einer Standardtherapie eingesetzt wird.

- Die Durchführung einer experimentellen Therapie ist mit erhöhten Sorgfaltspflichten verbunden.

Die Richtlinien verzichten bewusst auf eine Konkretisierung für einzelne Fachbereiche; sie enthalten aber in den ausführlichen Empfehlungen entsprechende Hinweise. Im Rahmen des vom Senat im November 2013 beschlossenen «Change-ControlProgramms» wird die Umsetzung einzelner Empfehlungen nachverfolgt. 


\section{Abgrenzung von Standardtherapie und experimenteller Therapie im Einzelfall*}

\section{Präambel}

Die Medizin befindet sich in einem stetigen Entwicklungsprozess. Ergebnisse aus der Qualitätssicherung und Forschung führen $\mathrm{zu}$ neuen Erkenntnissen und tragen zum Fortschritt bei. Was heute als Standardtherapie gilt, kann morgen überholt sein. Weil die Bewertung durch Expertinnen [1] und die Ausarbeitung fachlicher Empfehlungen Zeit brauchen, ist es möglich, dass eine Therapie für eine gewisse Zeit noch nicht als Standardbehandlung gilt, obwohl neueste wissenschaftliche Erkenntnisse dies nahelegen. Ältere Therapien hingegen gelten immer noch als Standard, obwohl neuere Daten und Erfahrungen sie obsolet erscheinen lassen.

Neuartige Behandlungen und Verfahren werden in der Regel im Rahmen von Forschungsprojekten wissenschaftlich evaluiert. Eine neuartige Behandlung kann aber auch unabhängig von der Durchführung eines Forschungsprojekts, aufgrund einer Therapieentscheidung bei einer einzelnen Patientin, zur Anwendung kommen; man spricht dann von einer «experimentellen Therapie im Einzelfall» [2].
Während Nutzen und Risiken der Standardtherapie im Allgemeinen bekannt sind, können Nutzen und Risiken einer experimentellen Therapie weniger gut abgeschätzt werden. Ob im Einzelfall eine Standardtherapie oder eine experimentelle Therapie zur Anwendung kommt, ist deshalb sowohl für die Patientin als auch für die behandelnde Ärztin von zentraler Bedeutung. Die Ärztin muss nachvollziehbare Gründe für den Einsatz einer experimentellen Therapie haben, und nur die Patientin selbst kann entscheiden, ob sie eine Therapie auf sich nehmen will, die mit unklaren Risiken verbunden ist. Dies setzt eine angemessene Information der Patientin voraus. Obwohl es selbstverständlich zu sein scheint, dass jeder medizinische Eingriff die «informierte Zustimmung» der Patientin braucht, zeigt die Praxis, dass eine genügende Aufklärung über den experimentellen Charakter einer Therapie nicht immer gewährleistet ist.

Die Schweizerische Stiftung SPO Patientenschutz forderte deshalb eine Regelung der sogenannten «Heilversuche» im Rahmen des Bundesgesetzes über Forschung am
Menschen [3]. Das Parlament hat dies abgelehnt, anerkennt jedoch, dass ein Bedarf nach mehr Klarheit über die verschiedenen Begrifflichkeiten besteht. Mit einer Motion hat es den Bundesrat beauftragt, die für den Bereich der Heilversuche geltenden Bestimmungen in der Schweiz aufzuzeigen, rechtliche Graubereiche zu erfassen, den Handlungsbedarf zu bestimmen und gegebenenfalls Vorschläge zur sachgerechten Ergänzung geltender Bestimmungen vorzulegen [4]. Die vorliegenden Richtlinien [5] definieren die Begriffe "Standardbehandlung» und «experimentelle Therapie im Einzelfall» und informieren über die bei der Anwendung von experimentellen Therapien im Einzelfall ausserhalb von Forschungsprojekten zu beachtenden Rechte und Pflichten von Ärztinnen und Patientinnen.

\section{Richtlinien}

\section{Geltungsbereich}

Die Richtlinien gelten für die gesamte Bandbreite medizinischer [6] Therapien, die im Rahmen einer Therapieentscheidung im

Tabelle 1

Ebenen der Standardisierung

\begin{tabular}{|c|c|c|c|c|c|c|}
\hline \multirow[b]{2}{*}{ Medizinische Evidenz } & \multicolumn{5}{|l|}{ Standardtherapie } & Experimentelle Therapi \\
\hline & $\begin{array}{l}\text { systematisches } \\
\text { Review mehrerer } \\
\text { randomisierter } \\
\text { kontrollierter Studien }\end{array}$ & $\begin{array}{l}\text { mindestens eine } \\
\text { randomisierte } \\
\text { kontrollierte Studie }\end{array}$ & $\begin{array}{l}\text { mehrere gut } \\
\text { kontrollierte Studien } \\
\text { ohne Randomisation }\end{array}$ & $\begin{array}{l}\text { publizierte } \\
\text { Fallserien }\end{array}$ & $\begin{array}{l}\text { eigene } \\
\text { Erfahrungen }\end{array}$ & präklinische Daten \\
\hline $\begin{array}{l}\text { Fachliche Empfehlungen } \\
\text { durch }\end{array}$ & $\begin{array}{l}\text { nationale oder } \\
\text { internationale } \\
\text { Fachgesellschaften }\end{array}$ & $\begin{array}{l}\text { strukturierter } \\
\text { Konsensus } \\
\text { repräsentativer } \\
\text { Expertinnengruppen }\end{array}$ & $\begin{array}{l}\text { informelle Expertin- } \\
\text { nengruppe }\end{array}$ & $\begin{array}{l}\text { einzelne } \\
\text { Expertinnen }\end{array}$ & eigene Ideen & \\
\hline Anwendungsformen & $\begin{array}{l}\text { Anwendung gemäss } \\
\text { Fachinformation }\end{array}$ & «off label use» & $\begin{array}{l}\text { «unlicensed use» } \\
\text { in der Schweiz }\end{array}$ & $\begin{array}{l}\text { «unlicensed use» } \\
\text { international }\end{array}$ & & \\
\hline $\begin{array}{l}\text { Kostenübernahme durch } \\
\text { obligat. Krankenpflege- } \\
\text { versicherung }\end{array}$ & generell & auf Gesuch & ausgeschlossen & & & \\
\hline Anwendungsqualität & $\begin{array}{l}\text { Routine mit } \\
\text { Qualitätssicherung }\end{array}$ & Routine & seltene Anwendung & $\begin{array}{l}\text { Erstmalige } \\
\text { Anwendung durch } \\
\text { behandelnde Ärztin }\end{array}$ & $\begin{array}{l}\text { erstmalige } \\
\text { bekannte } \\
\text { Anwendung }\end{array}$ & \\
\hline & 75,2 & 82,6 & 7,4 & 77,7 & 4,9 & \\
\hline
\end{tabular}

Die schematische Darstellung beschreibt den Übergang von Standardtherapie zur experimentellen Therapie im Einzelfall jeweils bezogen auf die einzelne Betrachtungsebene. Die Abstufungen der Standardisierung innerhalb der einzelnen Betrachtungsebenen stehen nicht in direktem Zusammenhang zueinander. 
Einzelfall zur Anwendung kommen. Dies umfasst präventive, diagnostische, therapeutische, palliative und rehabilitative Massnahmen, insbesondere in den Bereichen der Arzneimitteltherapien, der medizintechnischen Interventionen und Verfahren sowie der operativen Eingriffe.

Nicht in den Geltungsbereich der Richtlinien fallen medizinische Behandlungen, die im Rahmen von Forschungsprojekten durchgeführt werden.

\section{Ebenen der Standardisierung}

Eine klare und eindeutige Umschreibung von «Standardtherapie» existiert nicht. In der Rechtsprechung und Literatur wird die Standardtherapie in der Regel als «die Vorgehensweise, die dem aktuell geltenden Stand des Wissens entspricht», umschrieben. Wann im praktischen Alltag von Standardtherapie gesprochen wird, hängt aber unter anderem von der Ebene der Betrachtung ab (Tabelle 1). Der Grad der Evidenz für ihre zu erwartende Wirksamkeit, das Vorliegen von Empfehlungen für ihre Anwendung, die Existenz regulatorischer Bestimmungen der Heilmittelbehörde, die Kostenübernahme durch die Sozialversicherung und die Verfügbarkeit von Qualitätsstandards für ihre Anwendung können je im Rahmen unterschiedlicher Kontexte ausschlaggebend sein dafür, ob von einer Standardbehandlung gesprochen wird. Damit eine Verständigung möglich ist, muss eine einheitliche Betrachtungsebene gewählt werden.

\subsection{Medizinische Evidenz}

Der für eine medizinische Massnahme verfügbare Wirksamkeitsnachweis, wird nach den Regeln der Evidence-based Medicine (EBM) in Evidenzgrade (levels of evidence) eingeteilt. Dafür gibt es viele Klassifikationssysteme [7], die in unterschiedlicher Weise die Evidenz in Stufen vom höchsten Niveau systematischer Reviews von randomisierten Studien bis hinunter zu Fallserien mit historischen Kontrollen und pathophysiologischen Überlegungen einteilen. Für die Beurteilung der Evidenz im Hinblick auf die Erarbeitung von Guidelines wird statt der Verwendung einer einfachen Liste ein differenzierterer, situationsangepasster Prozess empfohlen (z.B. GRADE [8]).

Zur Ableitung von Behandlungsempfehlungen muss die bestmögliche verfügbare Evidenz kritisch bewertet werden. Dies erfordert ein hohes Mass an Fachkenntnis, klinischer Erfahrung und Urteilskraft. Expertinnen können in der Bewertung der gleichen Evidenz differieren. Es ist zu beachten, dass es viele Therapien gibt, die sich im Laufe der Zeit etabliert haben, die ausschliesslich auf Fallserien und pathophysiologischen Überlegungen basieren und die nie im Rahmen von kontrollierten Studien systematisch untersucht wurden.

\subsection{Fachliche Empfehlungen}

Therapieempfehlungen können als offizielle Guidelines von nationalen oder internationalen Fachgesellschaften, als Resultat eines Konsensusprozesses ausgewiesener Expertinnen oder als Lehrmeinungen einzelner fachlicher Autoritäten vorliegen. Ihre Relevanz hängt von der Aktualität und Vollständigkeit der berücksichtigten Evidenz, der Kompetenz der beteiligten Expertinnen, gemessen an deren Leistungsausweis in Forschung und Lehre auf dem entsprechenden Gebiet, und der Autorität der sie mittragenden offiziellen Instanzen (z.B. nationale oder internationale Fachgesellschaften) ab. Ein Instrument zur Beurteilung der Qualität von Guidelines ist z.B. AGREE [9]. Soweit von nationalen oder internationalen Fachgesellschaften anerkannte Guidelines existieren, stecken diese den Rahmen der Standardtherapie ab. Die Praktikerin ist verpflichtet, deren Anwendbarkeit auf die konkrete klinische Situation zu prüfen. Falls sie eine ausserhalb eines solchen Empfehlungsrahmens liegende Behandlungsoption wählt, muss sie diese Wahl nachvollziehbar begründen können [10]. In Situationen, für die keine anerkannten Guidelines existieren, kann auch die allgemein geübte medizinische Praxis, wie sie z.B. in internen Richtlinien von Spitälern oder der Lehrmeinung anerkannter Expertinnen zum Ausdruck kommt, zur Festlegung der Standardtherapie herangezogen werden.

\subsection{Zulassung durch die Heilmittel-} behörde

Für die Zulassung von Arzneimitteln und Medizinprodukten durch das Schweizerische Heilmittelinstitut (Swissmedic) muss die Anbieterin darlegen, dass ihr Produkt sicher und wirksam ist. Der Prozess der Überprüfung durch die Behörde unterscheidet sich jedoch in wesentlicher Hinsicht vom Prozess der Erarbeitung von fachlichen Empfehlungen. Es kann deshalb weder davon ausgegangen werden, dass ein Vorgehen gemäss offizieller Fachinformation eines Produkts automatisch den geltenden fachlichen Empfehlungen entspricht, noch dass für einen «off-label» oder «unlicensed use» [11] das Gegenteil der Fall ist. Ein registriertes Medikament kann obsolet geworden sein, ohne dass es seine Zulassung verloren hat. Viele oft gebrauchte - und nicht nur komplementärmedizinische - Heilmittel sind wohl zugelassen, werden aber in fachlichen Empfehlungen nicht erwähnt oder als wenig wirksam bezeichnet, sodass die Zulassung allein noch nicht eine Standardtherapie definiert. Umgekehrt sind verschiedene Gebiete der Medizin mit kleinem Markt, wie z. B. Pädiatrie, Geburtshilfe, Palliativmedizin und Intensivmedizin, gezwungen, viele fachlich empfohlene Behandlungen off-label durchzuführen, weil die Anwendung für diese Situationen (noch) nicht registriert ist bzw. aus wirtschaftlichen Überlegungen keine Registrierung angestrebt wird.

\subsection{Kostenübernahme durch die soziale Krankenversicherung}

Der Entscheid für die Kostenübernahme durch die soziale Krankenversicherung erfolgt über einen weiteren behördlichen Prozess, der sich auf Zulassungsinformationen, fachliche Empfehlungen und die Beurteilung durch speziell dafür ernannte Expertinnen abstützt. So ist bei Arzneimitteln die $\mathrm{Zu}$ lassung durch das Heilmittelinstitut eine Voraussetzung für die Aufnahme in die Spezialitätenliste (SL), die vom Bundesamt für Gesundheit geführt wird. Arzneimittel, die in der SL figurieren, werden in allen von Swissmedic zugelassenen Indikationen von der sozialen Krankenversicherung übernommen, sofern keine Einschränkung in der SL besteht. Die ausnahmsweise Kostenübernahme einer Therapie im «unlicensed» oder «off-label-use» im Einzelfall ist unter bestimmten Voraussetzungen (z.B. Orphan Diseases) möglich.

\subsection{Anwendungsqualität}

Medizinische Leistungserbringer sehen sich in den letzten Jahren zunehmend mit Forderungen nach Standardisierung ihrer Behandlungen im Hinblick auf Qualitätssicherung und Patientensicherheit konfrontiert. Qualitätsstandards werden dabei vorwiegend für häufig durchgeführte Massnahmen erarbeitet und implementiert. Damit erhält der Begriff Standardbehandlung die zusätzliche Dimension der Anwendungsqualität. Fachliche Empfehlungen beurteilen eine medizinische Massnahme primär unabhängig von der konkreten Situation ihrer Anwendung, allenfalls stellen sie gewisse Minimalanforderungen an die Qualifikation von Anwenderin und Institution. Die Anwendungsqualität im Einzelfall kann hingegen unterschiedlich sein. Ob eine Leistungserbringerin eine Massnahme zum ersten Mal selbstständig durchführt oder ob sie diese 
im Rahmen eines aktiv bearbeiteten Qualitätsstandards als Routine erbringt, kann zwar für die Patientin relevant sein, hat aber nichts mit der allgemeinen Beurteilung der Massnahme als solcher zu tun.

\section{Definitionen}

\subsection{Standardtherapie}

Aufgrund der Überlegungen in Kapitel 2 wird in diesen Richtlinien für die Definition der Standardbehandlung die Ebene der fachlichen Empfehlungen gewählt, weil sich diese am besten für die Beurteilung der Sorgfaltspflichten der Ärztin eignet. Entscheidend ist, ob die Ärztin sich mit einer Therapie im Bereich der aktuell anerkannten Empfehlungen bzw. der allgemein geübten Praxis bewegt oder ob sie diesen verlässt.

«Standardtherapie» beinhaltet für diese Richtlinien alle medizinischen Behandlungen, die sich auf aktuell gültige fachliche Empfehlungen (vgl. Kap. 2.2.) stützen oder beim Fehlen solcher Empfehlungen - der allgemein geübten Praxis anerkannter Expertinnen entsprechen.

\subsection{Experimentelle Therapie im Einzelfall}

Experimentelle Therapie im Einzelfall bedeutet für diese Richtlinien eine Therapie, die von der Standardtherapie abweicht bzw. die bei Fehlen einer Standardtherapie eingesetzt wird.

Die graduelle Verbesserung therapeutischer oder diagnostischer Verfahren im Rahmen von Qualitätsprojekten gilt nicht als experimentelle Therapie im Einzelfall, solange keine qualitativ neuen Schritte mit experimentellem Charakter eingefügt werden. Hingegen kann die Kombination von zwei Standardtherapien experimentellen Charakter aufweisen.

\subsection{Behandlung im Rahmen eines Forschungsprojekts}

Sowohl eine Standardtherapie als auch eine experimentelle Behandlung kann im Rahmen eines Forschungsprojekts angewendet werden. Forschungsuntersuchungen stellen nicht einen zusätzlichen Anteil des Behandlungsspektrums neben Standard- und experimenteller Therapie dar, sondern erweitern die Therapie um die zusätzliche Dimension des wissenschaftlichen Erkenntnisgewinns [12].

Um eine Behandlung im Rahmen eines Forschungsprojekts handelt es sich, wenn diese im Rahmen einer wissenschaftlichen Fragestellung durchgeführt wird und damit auch der Gewinnung verallgemeinerbarer
Erkenntnisse dient. Dabei ist in der Regel mindestens eines der folgenden Merkmale erfüllt [13]:

- Daten mehrerer Patientinnen werden prospektiv gesammelt und ausgewertet.

- Die Art und Applikationsweise der Therapie ist nicht nur an den Bedürfnissen der Patientinnen ausgerichtet, sondern wird auch durch das Forschungsprojekt bestimmt.

- Es werden zusätzliche, für die Behandlung nicht notwendige Daten erhoben bzw. Untersuchungen durchgeführt (z.B. Pharmakokinetik).

\section{Vorgehen beim Einsatz experimen- teller Therapien}

\subsection{Sorgfaltspflichten}

Im Rahmen der Therapiefreiheit kann die Ärztin der Patientin eine experimentelle Therapie vorschlagen, sofern sie dafür gute Gründe namhaft machen kann.

Die Anforderungen an die ärztlichen Sorgfaltspflichten richten sich gemäss Rechtsprechung des Bundesgerichts [14] nach den Umständen im Einzelfall und sind nach folgenden Kriterien zu bemessen:

- Art der Behandlung;

- Risiken und Nutzen der Behandlung;

- Ermessensspielraum;

- Mittel und Zeit, die der Ärztin zur Verfügung stehen, sowie

- Leistungsfähigkeit [15] und Aus-, Weiterund Fortbildung der Ärztin.

Bei experimentellen Therapien bestehen nach Massgabe der zu erwartenden Risiken erhöhte Sorgfaltspflichten. Bei Therapien, die infolge eines fehlenden Wirksamkeitsnachweises nicht als Standardtherapie gelten können, aber aufgrund langjährigen, verbreiteten Gebrauchs als nur mit minimalen Risiken verbunden eingeschätzt werden, muss wenigstens geprüft werden, ob durch die Anwendung der Patientin nicht eine wirksame Standardtherapie vorenthalten wird.

Bevor die Ärztin eine experimentelle Therapie im Einzelfall vorschlägt, muss sie die Standardtherapie und die experimentelle Therapie vergleichen sowie den möglichen Nutzen und die potenziellen Risiken für die Patientin abschätzen und dokumentieren.

Existiert keine Standardtherapie, muss die experimentelle Therapie gegen eine Nichtbehandlung abgewogen werden. Bei experimentellen Therapien muss die Ärztin in besonderem Masse mit bisher unbekannten Risiken und Nebenwirkungen rechnen.
Sie muss ihre Risiko-Nutzen-Abwägung auf eine rational begründete, nachvollziehbare Hypothese stützen und die Anhaltspunkte, die sich aufgrund der verfügbaren medizinischen Erfahrungen und wissenschaftlichen Erkenntnisse ergeben, systematisch berücksichtigen. Es muss eine begründete Aussicht auf Heilung, Besserung, Linderung oder Prävention bestehen.

Sind die klinische Expertise bzw. die technischen Fähigkeiten im Hinblick auf den Behandlungserfolg zentral, z. B. bei einer experimentellen Operationsmethode [16] oder einer komplexen Behandlungsstrategie, muss die Behandlerin überprüfen, ob sie das Verfahren genügend beherrscht oder ob die Patientin an einen anderen Ort mit entsprechender Erfahrung überwiesen werden sollte.

Vor der Durchführung einer experimentellen Therapie im Einzelfall soll die Behandlungsoption durch ein Expertinnengremium (z.B. Tumorboard, interdisziplinäre Fallbesprechung) überprüft oder mindestens eine Zweitmeinung eingeholt werden, es sei denn, das Risiko wird als minimal [17] eingeschätzt. Die Beurteilung, ob die zur Verfügung stehenden (präklinischen und klinischen) Daten für den erstmaligen Einsatz genügend Sicherheit bieten, kann so durch Expertinnen mit interdisziplinärer Perspektive erfolgen. Das Resultat der Expertise muss dokumentiert werden.

Die Therapie muss abgebrochen werden, wenn sich abzeichnet, dass sie erfolglos ist oder das Risiko-Nutzen-Verhältnis nicht vertretbar ist. Deshalb müssen alle Vorkehrungen getroffen werden, dass eine lückenlose Überwachung der Patientin möglich ist und ohne Verzug die erforderlichen Massnahmen zu ihrem Schutz ergriffen werden können. Da Nebenwirkungen spät auftreten können, müssen geeignete Massnahmen zur Nachverfolgung getroffen werden.

Da die mit einer experimentellen Therapie gewonnene Erfahrung auch für andere Patientinnen nützlich sein kann, sofern sie dokumentiert und veröffentlicht wird, soll bei experimentellen Behandlungen im Einzelfall vorgängig geprüft werden, ob die Behandlung nicht im Rahmen eines laufenden oder neu zu lancierenden Forschungsprojekts durchgeführt werden kann [18]. Ist dies nicht möglich, sollen die gewonnenen Erfahrungen (insbesondere auch negative Erfahrungen) nach Möglichkeit in einem Case-Report veröffentlicht und/oder in einem Register erfasst werden.

Werden experimentelle Therapien systematisch bei mehreren Patientinnen durchge- 
führt, kommen die Bestimmungen zur Forschung mit Menschen zur Anwendung [19].

\subsection{Aufklärung der Patientin [20]}

Die Aufklärung soll in einer der Patientin angepassten Form erfolgen und alle Informationen enthalten, die nötig sind, um eine informierte Entscheidung zu treffen [21]. Falls in einer Notfallsituation die Zeit für eine umfassende Aufklärung fehlt, muss diese so bald wie möglich nachgeholt werden. Massstab des individuellen Informationsbedürfnisses ist jeweils die direkt betroffene Patientin. Sie bedarf insbesondere aller Informationen, die eine Abwägung zwischen Nutzen und Risiken ermöglichen. Bei der Aufklärung über eine experimentelle Therapie muss die Patientin verstehen, dass die möglichen Risiken und der potenzielle Nutzen unter Umständen schwer abschätzbar sind. Falls in der konkreten Situation eine Standardtherapie zur Verfügung stehen würde, muss die Ärztin zudem begründen, warum sie eine experimentelle Therapie im Einzelfall vorschlägt.

Im Aufklärungsgespräch über die vorgeschlagene experimentelle Therapie sind zudem folgende Punkte in für die Patientin verständlicher Form zu besprechen:

- der aktuelle Gesundheitszustand der Patientin sowie der anzunehmende weitere Verlauf der Erkrankung;

- die vorgeschlagene Behandlung;

- mögliche Risiken und Belastungen der Behandlung, wobei die Aufklärung über Risiken neben Aussagen über die Häufigkeit und Schwere möglicher Schäden insbesondere auch Aussagen zur Bedeutung des Risikos und seine Eintrittswahrscheinlichkeit für die individuelle Patientin umfassen sollte (unabhängig von der absoluten Häufigkeit);

- beim «off-label-use»: alle relevanten Informationen, die sich nicht auf der Packungsbeilage finden;

- $\quad$ Art, Umfang, Ablauf, Dauer, Wirkung sowie Dringlichkeit der vorgeschlagenen Behandlung;

- mögliche Behandlungsalternativen;

- das Recht, auf eine angemessene Bedenkfrist bis zur Erteilung der Einwilligung;

- das Recht, die Einwilligung zur Behandlung ohne Angabe von Gründen zu widerrufen;

- das Recht eine Zweitmeinung einzuholen (eventuell Überweisung in ein anderes Spital oder zu einer anderen Ärztin);

- allfällige finanzielle Folgekosten (z.B. Selbstbeteiligung bei medikamentösen oder chirurgischen Behandlungen) sowie
- über die an einem anderen Ort verfügbare grössere Erfahrung mit der Massnahme.

Das Aufklärungsgespräch muss in der Krankengeschichte dokumentiert und die relevanten Informationen der Patientin schriftlich abgegeben werden. Entscheidungshilfen (z. B. Zeichnungen, Tabellen, Diagramme usw.) können das Verständnis erleichtern. Die Patientin ist einzuladen, Fragen zu stellen, Vertrauenspersonen mitzubringen und Unklarheiten zu äussern. Das Aufklärungsgespräch soll ein wirkliches Verständnis der Tragweite der vorgeschlagenen Behandlung vermitteln.

\subsection{Einwilligung der Patientin [22]}

Von einer informierten Einwilligung spricht man, wenn folgende Voraussetzungen erfüllt sind:

- Die Patientin ist urteilsfähig.

- Sie ist in ausreichendem Mass aufgeklärt.

- Sie ist in der Lage, die Aufklärung und die Reichweite ihrer Einwilligung zu verstehen.

- Sie kann ihre Wahl unbeeinflusst von Zwang oder Manipulation treffen und äussern.

Die Einwilligung der Patientin muss schriftlich erfolgen, es sei denn die experimentelle Therapie bringt höchstens minimale Risiken mit sich. Die Ärztin, die die Therapie verantwortet, trägt auch die Verantwortung für die korrekte Aufklärung und die erfolgte Einwilligung der Patientin.

\section{Empfehlungen}

Die nachfolgenden Empfehlungen richten sich an unterschiedliche Adressatinnen; sie sollen die Umsetzung der vorliegenden Richtlinien unterstützen.

\section{An die Fachgesellschaften}

- Ausarbeitung und regelmässige Aktualisierung von fachlichen Empfehlungen mit dem Ziel, den Rahmen der geltenden Standardtherapien möglichst klar und umfassend $\mathrm{zu}$ beschreiben soweit dies im entsprechenden Fachgebiet sinnvoll und machbar ist.

- Ausarbeitung von spezifischen Richtlinien zu den Sorgfaltspflichten beim Einsatz von experimentellen Therapien. Dies betrifft insbesondere das Vorgehen bei neuartigen Operations- oder Interventionsmethoden, beim «compassionate use» [23] von nicht zugelassenen
Arzneimitteln und bei der Einführung neuer Medical Devices.

\section{An die Spitäler}

- Förderung der Einrichtung und des Unterhalts interdisziplinärer Expertinnengremien (multidisziplinäre Fallbesprechungen, z.B. «Tumorboards» in der Onkologie) zur Besprechung komplexer und schwieriger Therapieentscheide.

- Unterstützung und Schulung interessierter Ärztinnen (z.B. durch Clinical Trial Units), damit experimentelle Therapien im Rahmen von Forschungsprojekten durchgeführt werden können.

\section{An die politischen Entscheidungsträger}

- Lösung der Probleme bei der Finanzierung von Therapien [24]:

a) Behandlungen, die gemäss anerkannten fachlichen Empfehlungen bereits zum Standard gehören, sollen solidarisch finanziert werden, auch wenn es sich um «off-label-use» handelt. Allerdings muss bei bezüglich Nutzen und Risiken gleichwertigen Behandlungsalternativen die wirtschaftlichere bevorzugt werden.

b) Experimentelle Behandlungen sollen mindestens in dem Umfang von der sozialen Krankenversicherung übernommen werden, wie sie der Standardbehandlung von Patientinnen mit der gleichen Erkrankung entspricht.

- Angleichung der Zulassungssituation für Standardtherapien an die EU, damit weniger «off-label-use» und weniger Medikamentenimport aus dem Ausland notwendig sind.

- Gleichbehandlung von Prüfsubstanzen [25] zum «compassionate use» von Schweizer Unternehmen und von Firmen aus Ländern mit vergleichbarer Arzneimittelsicherheit [26].

- Schaffen von Anreizen zur Erlangung der ordentlichen Zulassung (Durchführung von Zulassungsstudien) für seltenere Indikationen.

\section{An die pharmazeutische Industrie}

- Fortführung und Ausbau der Anstrengungen zur Offenlegung von Studiendaten, da diese wichtige Information für die Durchführung oder Vermeidung experimenteller Therapien enthalten können.

- Durchführung von Studien mit breiten Einschlusskriterien, um effizienter Daten zur Sicherheit und Wirksamkeit in der breiten therapeutischen Anwendung zu erhalten. 
- Führen von Compassionate-Use-Programmen für Prüfsubstanzen mit vitalem Nutzenpotenzial.

\section{Anhang}

Der Anhang mit Glossar und einem zweiten erläuternden Schema ist zugänglich unter www.samw.ch $\rightarrow$ Ethik.

* Vom Senat der SAMW am 20. Mai 2014 genehmigt. Die deutsche Fassung ist die Stammversion.

\section{Anmerkungen}

1 Die entsprechenden Texte betreffen immer beide Geschlechter der genannten Personengruppen.

2 «Experimentelle Therapien im Einzelfall» werden teilweise auch als «individuelle Heilversuche», «Therapie- oder Behandlungsversuche», «neuartige Heilbehandlungen», «Neulandbehandlungen», «nicht etablierte Behandlungen» usw. bezeichnet. Die Begriffe «therapeutische Forschung» oder «Humanexperiment», die ebenfalls verwendet werden, umschreiben hingegen Forschungshandlungen. Die vorliegenden Richtlinien verwenden ausschliesslich den Begriff «experimentelle Therapie im Einzelfall».

3 Vgl. das SPO-Argumentarium vom 22. April 2010, «Lücken im Humanforschungsgesetz». Mit der Regelung von Heilversuchen soll Rechtssicherheit für Patientinnen und Ärztinnen geschaffen werden.

4 Vgl. die Motion «Heilversuche» vom 12. Januar 2011 (11.3001).

5 Die Richtlinien der SAMW richten sich an medizinische Fachpersonen (Ärztinnen, Pflegefachpersonen und Therapeutinnen). Mit Aufnahme in die Standesordnung der FMH werden die Richtlinien für FMH-Mitglieder verbindliches Standesrecht.

6 Der Begriff «medizinisch» wird nachfolgend umfassend verwendet und bezieht sich auf die Tätigkeit von Ärztinnen, Pflegefachpersonen und Therapeutinnen.

7 Vgl. Oxford Centre for Evidence-based Medicine (www.cebm.net) oder Cochrane Deutschland (www.cochrane.de).

\section{Hinweise zur Ausarbeitung dieser Richtlinien}

\section{Auftrag}

Am 27. April 2011 hat die Zentrale Ethikkommission der SAMW eine Subkommission mit der Ausarbeitung von Richtlinien zur «nichtetablierten Behandlung von Patientinnen und Patienten im Einzelfall» beauftragt.

\section{Verantwortliche Subkommission}

Prof. Dr. med. Dieter Conen, Innere Medizin, Aarau (Vorsitz)

Prof. Dr. med. Nicolas Demartines, Chirurgie, Lausanne

Prof. Dr. med. Richard Herrmann, Onkologie, Riehen

Prof. Dr. med. Irene Hösli, Geburtshilfe, Basel
8 www.gradeworkinggroup.org

9 Vgl. The AGREE Collaboration. Appraisal of Guidelines for Research \& Evaluation (AGREE) Instrument 2010.

10 Vgl. Abteilung Daten, Demographie und Qualität (DDQ). Guidelines - Qualitätsmerkmale erkennen. Grundlagenpapier. Schweiz. Ärztezeitung 2014;95(3):45-51; sowie Meinung der FMH. Medizinische Guidelines: Voraussetzung und Anwendung. Schweiz Ärztezeitung. 2014;95(3):52-3.

11 Vgl. Glossar im Anhang.

12 Vgl. schematische Darstellung «Forschung» im Anhang.

13 Vgl. Art. 3 im Bundesgesetz über die Forschung vom 30. September 2011 und Botschaft zum Gesetzesentwurf vom 21. Oktober 2009.

14 Vgl. z.B. die Bundesgerichtsentscheide 134 IV 175, E. 3.2; 130 IV 7, E. 3.3; 120 II, E.2; 113 II 429, E. 3a.

15 Mit der (persönlichen) Leistungsfähigkeit einer Ärztin ist ihre eigene Erfahrung, ihr persönliches Wissen gemeint (z.B. wie oft sie einen Eingriff vorgenommen hat, in welchen Einrichtungen unter welchen Expertinnen sie die Ausbildung gemacht hat, ob sie in die Entwicklung einer neuen Methode persönlich involviert war oder nur darüber gelesen hat usw.). Je nachdem wie sich der Erfahrungsschatz und das persönliche Wissen einer Ärztin gestalten, kann von ihr eine höhere Sorgfalt verlangt werden. Umgekehrt ist die weniger erfahrene Ärztin unter Umständen verpflichtet, einen Eingriff an eine erfahrene Kollegin zu delegieren (bzw. sich Hilfe zu holen), da ihr das nötige Wissen und die Erfahrung (noch) fehlen.

16 Anwendung einer Operationsmethode, die in einigen Zentren bereits eingesetzt wird, sich jedoch noch nicht als Standard etabliert hat (early adoption)

17 «Minimal» umschreibt sowohl die Eintretenswahrscheinlichkeit eines Risikos als auch dessen Schweregrad.

18 Vgl. die Helsinki-Deklaration § 35: «In the treatment of a patient, where proven interventions do not exist or have been ineffective, the physician, after seeking expert

Prof. Dr. med. Christian Kern, Anästhesiologie, Lausanne

Margrit Kessler, Nationalrätin, Stiftung SPO Patientenschutz, Zürich

Prof. Dr. med. Christian Kind, ZEK-Präsident, Pädiatrie, St. Gallen

lic. iur. Michelle Salathé, SAMW, Recht, Basel Elisabeth Spichiger, PhD, RN, Pflegewissenschaft, Bern

Dr. iur. Franziska Sprecher, Recht, Zug

Prof. Dr. iur. utr. Brigitte Tag, Recht, Zürich

lic. iur. Barbara Züst, Stiftung SPO Patientenschutz, Winterthur

\section{Beigezogene Expertinnen}

Dr. sc. nat. Peter Brauchli, Dr. med. Hanne Hawle, SAKK, Bern

Prof. Dr. iur. Christian Brückner, Basel advice, with informed consent from the patient or a legally authorized representative, may use an unproven intervention if in the physician's judgement it offers hope of saving life, re-establishing health or alleviating suffering. Where possible, this intervention should be made the object of research, designed to evaluate its safety and efficacy. In all cases, new information should be recorded and, where appropriate, made publicly available.»

19 Vgl. Bundesgesetz über die Forschung am Menschen vom 30. September 2011.

20 Bei Patientinnen, die nicht urteilsfähig sind, entscheiden die gesetzlichen Vertretungspersonen. Das Gesetz erklärt folgende Personen bei medizinischen Massnahmen als vertretungsberechtigt. Die Reihenfolge ist hierarchisch: In erster Linie Personen, die in einer Patientenverfügung oder in einem Vorsorgeauftrag bezeichnet wurden, in zweiter Linie der Beistand mit einem Vertretungsrecht bei medizinischen Massnahmen, danach Angehörige und weitere Bezugspersonen, die der Patientin regelmässig persönlich Beistand leisten (Ehegatte bzw. eingetragener Partner, Personen im gleichen Haushalt, Nachkommen, Eltern, Geschwister). Für minderjährige Patientinnen sind die Inhaber der elterlichen Sorge vertretungsberechtigt. Die Vertretungsberechtigten sind analog aufzuklären.

21 Es ist zu beachten, dass in bestimmten Bereichen Aufklärungsinhalte und -formalitäten gesetzlich vorgegeben sind.

22 Bei Patientinnen, die nicht urteilsfähig sind, entscheiden die gesetzlichen Vertretungspersonen (vgl. Anmerkung 20). Diese sind analog aufzuklären.

23 Vgl. Glossar im Anhang.

24 Dies soll analog für Therapien gelten, die im Rahmen von Forschungsprojekten zur Anwendung kommen.

25 Als Prüfsubstanz werden pharmakologische Substanzen bezeichnet, die sich noch in der Phase der Überprüfung befinden und auch von der europäischen (EMA) oder amerikanischen (FDA) Arzneimittelbehörde nicht als Arzneimittel zugelassen sind.

26 Analog zur Regelung des Imports von in solchen Ländern zugelassenen Medikamenten.

Prof. Dr. med. Urs Haller, Appenzell Dipl. pharm. Annette Magnin, SCTO, Basel Prof. Dr. med. Felix Niggli, Isabelle Lamontagne MSc, SPOG, Bern

\section{Vernehmlassung}

Am 28. November 2013 hat der Senat der SAMW eine erste Fassung dieser Richtlinien genehmigt zur Vernehmlassung bei Fachgesellschaften, Organisationen und interessierten Personen. Die eingegangenen Stellungnahmen sind in der Endfassung berücksichtigt.

\section{Genehmigung}

Die definitive Fassung dieser Richtlinien wurde am 20. Mai 2014 vom Senat der SAMW genehmigt. 